\title{
Statistical Properties of the Periodogram for Stable Random Field
}

\author{
M.A. Ghazal* \\ Department of Mathematics, Faculty of Science, Mansoura University, New Damietta, Egypt
}

\begin{abstract}
Let $X(t)(t \in Z)$ be a discrete stable random field. The problem of estimating the spectral density field based on $X(t)$ is considered. Moments and the asymptotic moments of the spectral sample, the periodogram, based on $X(t)$ are calculated.
\end{abstract}

Keywords: Stable distributions, stationary stable processes, spectral representation, symmetric stable distribution, stable random field, periodogram.

\section{INTRODUCTION}

Paul Levy in the 1920s began the study of general stable distributions. He was interested in stable distributions because they are precisely the limit distributions that can occur in the Generalized Central Limit Theorem. A lot of works on stable distributions and related topics have been done for last two decades, see [1] and the references therein. Besides to the uses of stable distributions in probability and statistics, they have a wide applications in many fields Zolotarev [2], Ghazal [3-5], Combanis [6] and Hosoya [7].

Spectral analysis is an important technique in the statistical analysis. So, spectral representation of symmetric stable processes have been considered by Hardine [8]. Moreover, Masry [9] is concerned with the estimation of the spectral density for stationary stable processes. Therefore, we consider an estimation for the spectral density field of homogeneous symmetric complex $(n ; 1, \alpha)$ - stable field.

The paper is organized as follows: Section 2 is devoted to introduce some basic definitions and Lemmas which will be used later. In section 3, we are going to investigate the first and second moments of a periodogram for stable random fields. In section 4,the asymptotic moments of a periodogram for stable random fields will be given.

\section{PRELIMINARIES}

Definition (2.1): A complex random variable $Z=Z_{1}+i Z_{2}$ where $Z_{1}, Z_{2} \in R$, has a symmetric stable distribution with $\alpha$, $0<\alpha \leq 2$, if $Z_{1}$ and $Z_{2}$ have the same distribution and symmetric stable with $\alpha$.

Definition (2.2): A random field $\xi(\lambda), \lambda \in R^{\mathrm{n}}$, is called $(n ; 1$, $\alpha$ ) continuous stable random field if the linear combination $\sum_{k=1}^{m} Z_{k} \xi\left(\lambda^{(k)}\right)$ has a symmetric complex stable random field with where $Z_{k} \in \subset$ (the set of complex numbers); $\lambda^{(k)} R^{n}$ and $0<\alpha<2$.

*Address correspondence to this author at the Deptartment of Mathmatics, Faculty of Science, Mansoura University, New Damietta, Egypt; E-mail: maghazal@yahoo.com

AMS 1980 subject classification; primary 60G10, 60G99.
Definition (2.3): An $(n ; 1, \alpha)$ - stable random field $X(t)$ will be called discrete if $t \in Z^{n}$.

Definition (2.4): A random $(n ; 1, \alpha)$ - stable random field, $\xi(\lambda), \lambda \in R^{n}$, will be called homogeneous if all limiting distribution, $\xi(\lambda)=^{d} \xi(\lambda+\tau), \tau \in Z^{n}$ where $=^{d}$ (homogeneity of random field is defined by the translation-invariance of the finite-dimensional distribution.

Definition (2.5): Let $\xi(\lambda), \lambda \in \Pi^{n}$ be a continuous homogeneous symmetric complex $(n ; 1, \alpha)$ - stable random field with independent $(n ; 1, \alpha)$ - stable increment. A complex $(n ; 1, \alpha)$ - stable randon field $\eta(v), v R^{n}$, called harmonic homogeneous stable field if it has the spectral representation

$$
\eta(v)=\int_{R^{n}} \exp (i\langle v, \lambda\rangle d \xi(\lambda) .
$$

The spectral representation for discrete harmonic $(n ; 1$, $\alpha)$ - stable random field $X(t)$ can be written in the form

$$
X(t)=\int_{\Pi^{n}} \exp (i\langle v, \lambda\rangle d \xi(\lambda)
$$

where, $\xi(\lambda), \lambda \in \Pi^{n}$, is an $(n ; 1, \alpha)$ - stable random field with independent increments satisfy

$$
\left[E|d \xi(\lambda)|^{P}\right]^{\frac{\alpha}{P}}=C(P, \alpha) \Phi(\lambda) d \lambda
$$

where $\mathrm{C}(\mathrm{P}, \alpha)$ depends on $P$ and $\alpha$ and $\Phi(\lambda)$ is a nonnegative integrable function called the spectral density field of $X(t), t \in Z^{n}$.

We will construct an estimation for the nonnegative integrable function $\Phi(\lambda), \lambda \in \Pi^{n}$, on observations $X(t), t \in$ $Z^{n}$. For construction, we will use the periodogram as in Masry [9] which estimated the spectral density function for stable random process. Let $h(t)=h\left(t_{1}, t_{2}, \ldots t_{n}\right)$ be a bounded even function. Let $T=\left(T_{1}, T_{2}, \ldots T_{\mathrm{n}}\right)$ where $T_{j}=2 \tau_{\mathrm{j}}+1 ; j=$ $\overline{1, n}$ and $t_{\tau}=\left(\frac{t_{1}}{\tau_{1}}, \frac{t_{2}}{\tau_{2}}, \cdots \cdots, \frac{t_{n}}{\tau_{n}}\right)$. We define a finite Fourier transform of the function $h(t)$ by 
$H^{(T)}(\lambda)=\sum_{t \in T^{n}} h\left(t_{\tau}\right) \exp (-i\langle t, \lambda\rangle)$, where $\lambda \in \prod^{n}$.

The function $H^{(T)}(\lambda)$ satisfy

$$
B_{\alpha}^{(T)}=\int_{\prod^{n}}\left|H^{(T)}(\lambda)\right|^{\alpha} d \lambda<\infty \text { with } 0<\alpha<2 .
$$

Let

$$
H_{T}(\lambda)=A_{T} H^{(T)}(\lambda)
$$

where $A_{T}=\left[\frac{1}{B_{\alpha}^{(T)}}\right]^{\frac{1}{\alpha}}, \lambda \in \prod^{n}$ and $\int_{\prod^{n}}\left|H_{T}(\lambda)\right|^{\alpha} d \lambda=1$.

We consider the statistic

$$
d_{T}(\lambda)=A_{T} \operatorname{Re}\left[\sum_{t \in T} \exp (-i\langle t, \lambda\rangle) h\left(t_{\tau}\right) X(t)\right]
$$

to estimate the spectral density $\Phi(\lambda)$.

Definition (2.6): Let $0<P<\alpha \leq 2, \lambda \in \Pi^{n}$. The statistic $I_{T}(\lambda)$ will be called a periodogram $(n ; 1, \alpha)$ - stable random field $X(t), t \in T^{n}$, where

$$
\begin{aligned}
& I_{T}(\lambda)=K(P, \alpha)\left|d_{T}(\lambda)\right|^{P}, \\
& K(P, \alpha)=\frac{D(P)}{F(P, \alpha) C_{\alpha}^{P / \alpha}},
\end{aligned}
$$

with

$$
F(P, \alpha)=\int_{-\infty}^{\infty} \frac{1-e^{-|u|^{\alpha}}}{|u|^{1+P}} d u
$$

The following Lemma can be proved as in [9] and will be used in Section 3.

Lemma (2.1): Let $\mathrm{L}^{\alpha}(0<\alpha \leq 2)$ be the set of all measurable function on $\Pi^{n}$ for which $\int_{\Pi^{n}}|g(\lambda)|^{\alpha} d G(\lambda)<\infty$, where $\prod^{n}$

$G(\lambda)$ is non-negative bounded on $\Pi^{n}$ with $G(\Pi, \Pi, \ldots, \Pi)>0$.

Then for homogeneous symmetric complex $(n ; 1, \alpha)$ - stable field $\xi(\lambda), \lambda \in \prod^{n}$, we have

$$
\left.\left.E \exp \left\{i \operatorname{Re} \int_{\prod^{n}} g(\lambda) d \xi(\lambda)\right]\right\}=\int_{\prod^{n}}|g(\lambda)|^{\alpha} \Phi(\lambda) d \lambda\right]
$$

where $C_{\alpha}=\frac{1}{\pi} \int_{0}^{\pi}|\cos \theta|^{\alpha} d \theta$.

The following Lemmas which was proved by Masry [9] will be used in the sequel.

Lemma (2.2): $\operatorname{IF} \mathrm{D}(P)=\int_{-\infty}^{\infty} \frac{1-\cos u}{|u|^{1+P}}$, then

$$
|x|^{P}=D^{-1}(P) \int_{-\infty}^{\infty} \frac{1-\cos (x u)}{|u|^{1+P}} d u=D^{-1}(P) \operatorname{Re}\left[\int_{-\infty}^{\infty} \frac{1-e^{i x u}}{|u|^{1+P}} d u\right] .
$$

Lemma (2.3): If $x, y \in R$ with $0<\alpha \leq 2$, then

$$
\| X+\left.y\right|^{\alpha}-|X|^{\alpha}-\left.|y|^{\alpha}|\leq 2| x y\right|^{\frac{\alpha}{2}} .
$$

\section{MEAN, DESPERSION AND COVARIANCE FOR THE PERIODOGRAM}

In this section we will investigate the essential feature of the spectral representation of symmetric stable random field. This can be done by considering the first and second-order moments of the periodogram $\operatorname{IT}(\lambda)$.

The following lemma rewrite $d_{T}(\lambda)$, in a useful form.

Lemma (3.1): For $\lambda \in \Pi^{n}$, the statistic $d_{T}(\lambda)$ satisfies

$$
d_{T}(\lambda)=\int_{\prod^{n}} H_{T}(\lambda-\mu) d \xi(\mu),
$$

where $\xi_{1}(\mu)=\operatorname{Re} \xi(\mu)$ and $\xi(\mu)$ uniform symmetric complex discrete $(n ; 1, \alpha)$ - stable field.

Proof. The proof can be accomplished from (2.4) and using (2.1), (2.2) and (2.3).

Lemma (3.2): For $a \in R, b \in \mathrm{R}, \lambda, v \in \Pi^{n}$, then

$$
\begin{aligned}
& E \exp \left[i\left(a d_{T}(\lambda)+b d_{T}(v)\right]\right. \\
& =\exp \left[-C_{\alpha} \int_{\prod^{n}}\left|a H_{T}(\lambda-\mu)+H_{T}(v-\mu)\right|^{\alpha} \Phi(\mu) d \mu\right]
\end{aligned}
$$

Proof: The proof can bededuced from Lemma (3.1) and Lemma (2.1).

The characteristic function of $d_{T}(\lambda)$ may be stated as follows:

Corollary (3.1): Let $\lambda \in \Pi^{n}$ and $0<\alpha \leq 2$. Then

$$
E \exp \left[i\left(a d_{T}(\lambda)\right]=\exp \left[-C_{a}|a|^{a} \gamma_{T}^{(\alpha)}(\lambda)\right.\right.
$$

where

$$
\left.\gamma_{T}^{(\alpha)}(\lambda)=\int_{\prod^{n}}\left|H_{T}(\lambda-\mu)\right|^{\alpha} \Phi(\mu) d u\right]
$$

Theorem (3.1): Let $\lambda \in \Pi^{n}$. Then

(i) $E I_{T}(\lambda)=\left[\gamma_{T}^{(\alpha)}(\lambda)\right]^{\frac{P}{\alpha}}, P \in(0, \alpha) \quad$ (3.4)

(ii) $D I_{T}(\lambda)=\left[\frac{K^{2}(P, \alpha)}{K(2 P, \alpha)}-1\right]\left[\gamma_{T}^{(\alpha)}(\lambda)\right]^{\frac{2 P}{\alpha}}, P \in\left(0, \frac{\alpha}{2}\right)$.

Proof: From (2.9), we have

$$
\left|d_{T}(\lambda)\right|^{\alpha}=D^{-1}(P) \operatorname{Re}\left[\int_{-\infty}^{\infty} \frac{1-\exp \left(i u d_{T}(\lambda)\right)}{|u|^{1+P}} d u\right]
$$

From (2.5), (3.2) and (2.6) we can obtain

$$
\left.E I_{T}(\lambda)=\frac{1}{F(P, \alpha) C_{\alpha}^{p / \alpha}} \int_{-\infty}^{\infty} \frac{1-\exp \left(C_{\alpha}|a|^{\alpha} \gamma_{T}^{(\alpha)}(\lambda)\right)}{|u|^{1+P}} d u\right]
$$

By putting $u=\frac{x}{\left[C_{\alpha} \gamma_{T}^{(\alpha)}(\lambda)\right]^{1 / \alpha}}$, and using (2.7) we have

$$
E I_{T}(\lambda)=\frac{1}{F(P, \alpha)} \int_{-\infty}^{\infty} \frac{1-\exp \left(-|x|^{\alpha}\right)}{|x|^{1+P}} d u\left[\gamma_{T}^{(\alpha)}(\lambda)\right]^{\frac{P}{\alpha}} \text {. }
$$


Part (ii) can be proved by a similar way.

\section{Theorem (3.2):}

$\left.\operatorname{Cov}\left\{I_{T}\left(\lambda^{(1)}\right), \operatorname{IT}\left(\lambda^{(2)}\right)\right)\right\}=$

$$
\begin{aligned}
& =\left[\frac{1}{F(P, \alpha) C_{\alpha}^{P / \alpha}}\right]^{2} \iint_{-\infty}^{\infty}\left[\exp \left\{C_{T}^{(1)}\left(u_{1}, u_{2}\right)\right\}\right. \\
& \left.-\exp \left\{C_{T}^{(2)}\left(u_{1}, u_{2}\right)\right\}\right] \frac{d u_{1} d u_{2}}{\left|u_{1} u_{2}\right|^{1+P}}
\end{aligned}
$$

where

$$
\begin{aligned}
& C_{T}^{(1)}\left(u_{1}, u_{2}\right)=-C_{\alpha} \int_{\prod^{n}} u_{1} H_{T}\left(\lambda^{(1)}-\mu\right) \\
& +\left.u_{2} H_{T}\left(\lambda^{(2)}-\mu\right)\right|^{\alpha} \Phi(\mu) d \mu \\
& C_{T}^{(1)}\left(u_{1}, u_{2}\right)=-C_{\alpha} \int_{\prod^{n}} u_{1} H_{T}\left(\lambda^{(1)}-\mu\right) \\
& \left.+\left|u_{2} H_{T}\left(\lambda^{(2)}-\mu\right)\right|^{\alpha}\right) \Phi(\mu) d \mu
\end{aligned}
$$

Proof: From (2.5),(2.6) and (2.9) we have

$$
I_{T}(\lambda)=\frac{1}{F(P, \alpha) C_{\alpha}^{P / \alpha}} \int_{-\infty}^{\infty} \frac{1-\cos \left(u d_{T}(\lambda)\right)}{|u|^{1+P}} d u
$$

From (3.6) we obtain

$$
=\frac{1}{F(P, \alpha) C_{\alpha}^{P / \alpha}} \int_{-\infty}^{\infty}\left[\cos \left(u d_{T}(\lambda)\right)+\exp \left(-C_{\alpha}|u|^{\alpha} \gamma_{T}^{(\alpha)}(\lambda)\right)\right] \frac{d u}{|u|^{1+P}}
$$

By the definition of the covariance function, we can obtain

$$
\begin{aligned}
& \operatorname{Cov}\left\{I_{T}\left(\lambda^{(1)}\right), I_{T}\left(\lambda^{(2)}\right)\right\}=\left[\frac{1}{F\left(P, \alpha_{-} C_{\alpha}^{P / \alpha}\right.}\right]^{2} \iint_{-\infty}^{\infty} E\left\{\prod _ { j = 1 } ^ { 2 } \left[-\cos \left(u_{j} d_{T}\left(\lambda^{(1)}\right)\right)\right.\right. \\
& \left.+\exp \left(-C_{\alpha}\left|u_{j}\right|^{\alpha} \gamma_{T}^{(\alpha)}\left(\lambda^{(1)}\right)\right)\right\} \frac{d u_{1} d u_{2}}{\left|u_{1} u_{2}\right|^{1+P}}
\end{aligned}
$$

Since,

$$
\begin{aligned}
& E\left\{\prod_{j=1}^{2}\left[-\cos \left(u_{j} d_{T}\left(\lambda^{(1)}\right)+\exp \left(-C_{\alpha}\left|u_{j}\right|^{\alpha} \gamma_{T}^{(\alpha)}\left(\lambda^{(j)}\right)\right)\right]\right\}\right. \\
& =E\left\{\cos \left(u_{1} d_{T}\left(\lambda^{(1)}\right)\right) \cos \left(u_{2} d_{T}\left(\lambda^{(2)}\right)\right)\right\} \\
& \quad-\exp \left(-C_{\alpha}\left|u_{2}\right|^{\alpha} \gamma_{T}^{(\alpha)}\left(\lambda^{(2)}\right)\right) E\left\{\cos \left(u_{1} d_{T}\left(\lambda^{(1)}\right)\right)\right\} \\
& \quad-\exp \left(-C_{\alpha}\left|u_{1}\right|^{\alpha} \gamma_{T}^{(\alpha)}\left(\lambda^{(1)}\right)\right) E\left\{\cos \left(u_{2} d_{T}\left(\lambda^{(2)}\right)\right)\right\} \\
& \left.\quad+\exp \left[-C_{\alpha}\left|u_{1}\right|^{\alpha} \gamma_{T}^{(\alpha)}\left(\lambda^{(1)}\right)+\left|u_{2}\right|^{\alpha} \gamma_{T}^{(\alpha)}\left(\lambda^{(2)}\right)\right)\right]
\end{aligned}
$$

From Lemma (3.2), we get

$E\left\{\cos \left(u_{1} d_{T}\left(\lambda^{(1)}\right) \cos \left(u_{2} d_{T}\left(\lambda^{(2)}\right)\right\}\right.\right.$

$$
\begin{aligned}
& =\frac{1}{2} \exp \left[-C_{\alpha} \int_{\prod^{n}}\left|u_{1} H_{T}\left(\lambda^{(1)}-\mu\right)+u_{2} H_{T}\left(\lambda^{(2)}-\mu\right)\right|^{\alpha} \Phi(\mu) d \mu\right] \\
& +\frac{1}{2} \exp \left[-C_{\alpha} \int_{\prod^{n}}\left|u_{1} H_{T}\left(\lambda^{(1)}-\mu\right)-u_{2} H_{T}\left(\lambda^{(2)}-\mu\right)\right|^{\alpha} \Phi(\mu) d \mu\right]
\end{aligned}
$$

Furthermore, by using (3.2) we get

$$
E\left\{\cos \left(u_{j} d_{T}\left(\lambda^{(1)}\right)\right)\right\}=\exp \left(-C_{\alpha}\left|u_{j}\right|^{\alpha} \gamma_{T}^{(\alpha)}\left(\lambda^{(j)}\right)\right)
$$

By subsituting from (3.11) and (3.12) in (3.10), then we have $\operatorname{Cov}\left\{I_{T}\left(\lambda^{(1)}\right), I_{T}\left(\lambda^{(2)}\right)\right\}$

$$
\begin{aligned}
& =\left[\frac{1}{F(P, \alpha) C_{\alpha}^{P(\alpha}}\right]^{2}\left[-\iint_{-\infty}^{\infty} \exp \left[-C_{\alpha}\left(\left|u_{1}\right|^{\alpha} \gamma_{T}^{(\alpha)}\left(\lambda^{(1)}\right)+\left|u_{2}\right|^{\alpha} \gamma_{T}^{(\alpha)}\left(\lambda^{(2)}\right)\right] \frac{d u_{1} d u_{2}}{\left|u_{1} u_{2}\right|^{1+P}}\right.\right. \\
& +\frac{1}{2} \iint_{-\infty}^{\infty} \exp \left[-C_{\alpha} \int_{\prod^{n}}\left|u_{1} H_{T}\left(\lambda^{(1)}-\mu\right)+u_{2} H_{T}\left(\lambda^{(2)}-\mu\right)\right|^{\alpha} \Phi(\mu) d \mu\right]\left(\frac{d u_{1} d u_{2}}{\left|u_{1} u_{2}\right|^{1+P}}\right. \\
& +\frac{1}{2} \iint_{-\infty}^{\infty} \exp \left[-C_{\alpha} \int_{\prod^{n}}\left|u_{1} H_{T}\left(\lambda^{(1)}-\mu\right)-u_{2} H_{T}\left(\lambda^{(2)}-\mu\right)\right|^{\alpha} \Phi(\mu) d \mu\right]\left(\frac{d u_{1} d u_{2}}{\left|u_{1} u_{2}\right|^{1+P}}\right.
\end{aligned}
$$

Finally, if we replace $u_{2}$ by $\left(-u_{2}\right)$ and using (3.3), (3.8), then the proof can be completed.

\section{ASYMPTOTIC BEHAVIOUR OF THE MEAN, DESPESION AND COVARIANCE FOR THE PERIO- DOGRAM}

In this section we obtain the asymptotic properties of the periodogram $I_{T}(\lambda)$.

Definition (4.1): A postive kernal $F_{T}(\lambda), \lambda \in \Pi^{n}$, is said to be $n$ - measure sequence function if for all $\lambda \in \Pi^{n}$ and $\delta>$ 0 , the following conditions are satisfied:

$$
\begin{aligned}
& F_{T}(\lambda) \geq 0 ; \\
& \int_{\prod^{n}} F_{T}(\lambda) d \lambda=1
\end{aligned}
$$

$$
\lim _{T \rightarrow \infty} \int_{\prod^{n} / S_{\delta}^{0}} F_{T}(\lambda) d \lambda=0
$$

where $S_{\delta}^{0}=\left\{\lambda=\left(\lambda_{1}, \lambda_{2}, \ldots \ldots, \lambda_{\mathrm{n}}\right),\|\lambda\|<\delta, \delta>0\right\}$

The following lemma will be used later in the proof of Theorem (4.1).

Lemma (4.1): Let $F_{7}(\lambda), \lambda \in \Pi^{n}$, be a positive kernal. If $g(\lambda)$ is bounded on $\Pi^{n}$ and continuous at a point $\lambda^{*}, \lambda^{*} \in \Pi^{n}$, then

$$
\lim _{T \rightarrow \infty} \int_{\prod^{n}} F_{T}\left(\lambda+\lambda^{*}\right) d \lambda=g\left(\lambda^{*}\right) .
$$

Proof: Since $\int_{\prod^{n}} F_{T}(\lambda+) d \lambda=1$, then

$\left|\int_{\prod^{n}} F_{T}(\lambda) g\left(\lambda+\lambda^{*}\right) d \lambda-g\left(\lambda^{*}\right)\right| \leq \mid$

Since $g(\lambda)$ continuous at $\lambda^{*}$ then for every $\varepsilon>0$ there exists $\delta>0$ such that $\left|g\left(\lambda+\lambda^{*}\right)-g\left(\lambda^{*}\right)\right| \leq \varepsilon$ for all $\lambda \in S_{\delta}^{0}$. So,

$$
\begin{aligned}
& \left|\int_{\prod^{n}} F_{T}(\lambda) g\left(\lambda+\lambda^{*}\right) d \lambda-g\left(\lambda^{*}\right)\right| \\
& \leq \varepsilon\left|\int_{S_{\delta}^{0}} F_{T}(\lambda) d \lambda\right|+\left|\int_{\prod^{n} / S_{\delta}^{0}} F_{T}(\lambda)\right| g\left(\lambda+\lambda^{*}\right)-g\left(\lambda^{*}\right)|d \lambda| \\
& \leq \varepsilon+\left|\int_{\prod^{n} / S_{\delta}^{0}} F_{T}(\lambda)\right| g\left(\lambda+\lambda^{*}\right)-g\left(\lambda^{*}\right)|d \lambda|
\end{aligned}
$$


Since $g(\lambda)$ is bounded on $\Pi^{n}$, then there is a real number $L<\infty$ such that ${ }_{\lambda \in} \prod^{\text {sup }}$.

Therefore,

$$
\int_{\prod^{n} / s_{\delta}^{0}} F_{T}(\lambda)\left|g\left(\lambda+\lambda^{*}\right)-g\left(\lambda^{*}\right)\right| d \lambda \leq 2 L \int_{\prod^{n} / S_{\delta}^{0}} F_{T}(\lambda) d \lambda
$$

According to definition (4.1) and $\left|g\left(\lambda+\lambda^{*}\right)-g\left(\lambda^{*}\right)\right|$ may be made arbitrarily small by choice of $\varepsilon$ as $g(\lambda)$ continuous at a point, we can conclude that

$\lim _{T x \rightarrow 0}\left[\int_{\prod^{n}} F_{T}(\lambda) g\left(\lambda+\lambda^{*}\right) d \lambda-g\left(\lambda^{*}\right)\right]=0$.

Theorem (4.1): Let $\lambda \in \Pi^{n}$. Then

(i) $\lim _{T \rightarrow \infty} E I_{T}(\lambda)=[\Phi(\lambda)]^{\frac{P}{\alpha}}, P \in(o, \alpha)$

(ii) $\lim _{T \rightarrow \infty} D I_{T}(\lambda)=\left[\frac{K^{2}(P, \alpha)}{K(2 P, \alpha)}-1\right][\Phi(\lambda)]^{\frac{2 P}{\alpha}}, P \in\left(0, \frac{\alpha}{2}\right)$.

Proof: The proof comes directly by substituting $-\lambda+\mu=v$ in (3.3) and using Theorem (3.1) with Lemma (4.1).

Theorem (4.2): Suppose $0 \mathrm{P} \leq \frac{\alpha}{2}, 0<\alpha \leq 2$ with $\lambda^{(1)} \in \Pi^{n}$, $\lambda^{(2)} \in \Pi^{n}$ and $\lambda_{j}^{(1)} \neq \lambda_{j}^{(2)}, j=\overline{1, n}$. Let $\Phi(\lambda), \lambda \in \Pi^{n}$, be continuous at $\lambda^{(1)}, \lambda^{(2)}$ and $\Phi\left(\lambda^{(1)}\right) \neq 0, \Phi\left(\lambda^{(2)}\right) \neq 0$. If

$\lim _{T \rightarrow \infty} \frac{B_{\alpha}^{(T)}\left(\lambda^{(1)}, \lambda^{(2)}\right.}{B_{\alpha}^{(T)}}=0$

where

$$
B_{\alpha}^{(T)}\left(\lambda^{(1)}, \lambda^{(2)}\right)=\int_{\prod^{n}}\left|H^{(T)}\left(\lambda^{(1)}-\lambda\right) H^{(T)}\left(\lambda^{(2)}-\lambda\right)\right|^{\alpha / 2} d \lambda
$$

Then

$$
\lim _{T \rightarrow \infty} \operatorname{Cov}\left\{I_{T}\left(\lambda^{(1)}\right), I_{T}\left(\lambda^{(2)}\right)\right\}=0
$$

Proof: From Theorem (3.2), we get

$$
\left|\operatorname{Cov}\left\{I_{T}\left(\lambda^{(1)}\right), I_{T}\left(\lambda^{(2)}\right)\right\}\right|
$$$$
\leq\left[\frac{1}{F(P, \alpha) C_{\alpha}^{\frac{p}{\alpha}}}\right] \iint_{-\infty}^{\infty}\left|C_{T}^{(1)}\left(u_{1}, u_{2}\right)-C_{T}^{(2)}\left(u_{1}, u_{2}\right)\right|
$$$$
\times \exp \left(\left|C_{T}^{(1)}\left(u_{1}, u_{2}\right)-C_{T}^{(1)}\left(u_{1}, u_{2}\right)\right|\right.
$$$$
\left.-C_{T}^{(1)}\left(u_{1}, u_{2}\right)\right) \frac{d u_{1} d u_{2}}{\left|u_{1} u_{2}\right|^{1+P}}
$$

From (3.8) and Lemma (2.3) we have

$$
\left|C_{T}^{(1)}\left(u_{1}, u_{2}\right)-C_{T}^{(2)}\left(u_{1}, u_{2}\right)\right| \leq 2 C_{\alpha}\left|u_{1}, u_{2}\right|^{\alpha / 2 \mu} \mu \prod^{\text {sup }} \Phi(\mu) \frac{B_{\alpha}^{(T)}\left(\lambda^{(1)}, \lambda^{(2)}\right)}{B_{\alpha}^{(T)}}
$$

Hence,
$\left|\operatorname{Cov}\left\{I_{T}\left(\lambda^{(1)}\right), I_{T}\left(\lambda^{(2)}\right)\right\}\right|$

$$
\begin{aligned}
& \leq\left[\frac{1}{F(P, \alpha) C_{\alpha}^{\frac{P}{\alpha}}}\right]_{\mu \in}^{2} \prod^{\text {sup }} \Phi(\mu) \frac{B_{\alpha}^{(T)}\left(\lambda^{(1)}, \lambda^{(2)}\right)}{B_{\alpha}^{(T)}} \iint_{-\infty}^{\infty} 2\left|u_{1} u_{2}\right|^{\frac{\alpha}{2}-(1+P)} \\
& \times \exp \left(\left|C_{T}^{(1)}\left(u_{1}, u_{2}\right)-C_{T}^{(2)}\left(u_{1}, u_{2}\right)\right|-C_{T}^{(2)}\left(u_{1}, u_{2}\right)\right) d u_{1} d u_{2}
\end{aligned}
$$

From Lemma (4.1), we obtain

$$
\lim _{T \rightarrow \infty} C_{T}^{(1)}\left(u_{1}, u_{2}\right)=\lim _{T \rightarrow \infty}\left(-C_{\alpha} \sum_{j=1}^{2}\left|u_{j}\right|{ }^{\alpha} \gamma_{T}^{(\alpha)}\left(\lambda^{(j)}\right)\right)=-C_{\alpha} \sum_{j=1}^{2} \mid u_{j}{ }^{\alpha} \Phi\left(\lambda^{(j)}\right)
$$

Also, by using (4.3) we have

$\lim _{T \rightarrow \infty}\left|C_{T}^{(1)}\left(u_{1}, u_{2}\right)-C_{T}^{(2)}\left(u_{1}, u_{2}\right)\right|=0$

Hence,

$\lim _{T \rightarrow \infty}\left|C_{T}^{(1)}\left(u_{1}, u_{2}\right)-C_{T}^{(2)}\left(u_{1}, u_{2}\right)\right|-C_{T}^{(2)}\left(u_{1}, u_{2}\right)=-C_{\alpha} \sum_{j=1}^{2}\left|u_{j}\right|^{\alpha} \Phi\left(\lambda^{(j)}\right)$

Therefore,

$\lim _{T \rightarrow \infty} \int_{-\infty}^{\infty} \int_{-\infty} u_{1},\left.u_{2}\right|^{\frac{\alpha}{2}-(1+P)} \exp \left(\left|C_{T}^{(1)}\left(u_{1}, u_{2}\right)-C_{T}^{(2)}\left(u_{1}, u_{2}\right)\right|\right.$

$\left.-C_{T}^{(2)}\left(u_{1}, u_{2}\right)\right) d u_{1} d u_{2}$

$=\prod_{j=1}^{2}\left[\int_{-\infty}^{\infty} \exp \left(-C_{\alpha}\left|u_{j}\right|^{\alpha} \Phi\left(\lambda^{(j)}\right)\right)\left|u_{j}\right|^{\frac{\alpha}{2}-(1+P)} d u_{j}\right]$

$\int_{-\infty}^{\infty} \exp \left(-C_{\alpha}\left|u_{j}\right|^{\alpha} \Phi\left(\lambda^{(j)}\right)\right)\left|u_{j}\right|^{\frac{\alpha}{2}-(1+P)} d u_{j}$

$=2\left[\int_{0}^{1} \exp \left(-C_{\alpha}\left|u_{j}\right|^{\alpha} \Phi\left(\lambda^{(j)}\right)\right)\left|u_{j}\right|^{\frac{\alpha}{2}-(1+P)} d u_{j}\right.$

$\left.+\int_{1}^{\infty} \exp \left(-C_{\alpha}\left|u_{j}\right|^{\alpha} \Phi\left(\lambda^{(j)}\right)\right)\left|u_{j}\right|^{\frac{\alpha}{2}-(1+P)} d u_{j}\right]$

For P $(0,2)$

$\int_{0}^{1} \exp \left(-C_{\alpha}\left|u_{j}\right|^{\alpha} \Phi\left(\lambda^{(j)}\right)\right)\left|u_{j}\right|^{\frac{\alpha}{2}-(1+P)} d u_{j} \leq \int_{0}^{1}\left|u_{j}\right|^{\frac{\alpha}{2}-(1+P)} d u_{j}=\frac{1}{\frac{\alpha}{2}-P}$

Also,

$$
\begin{aligned}
& \int_{1}^{\infty} \exp \left(-C_{\alpha}\left|u_{j}\right|^{\alpha} \Phi\left(\lambda^{(j)}\right)\right)\left|u_{j}\right|^{\frac{\alpha}{2}(1+P)} d u_{j} \\
& \leq \int_{1}^{\infty} \exp \left(-C_{\alpha}\left|u_{j}\right|^{\alpha} \Phi\left(\lambda^{(j)}\right)\right) d u_{j} \leq \frac{1}{C_{\alpha}\left(\lambda^{(j)}\right)}
\end{aligned}
$$

Therefore,

Finally, we can conclude that

$$
\begin{aligned}
& \lim _{T \rightarrow \infty} \iint_{-\infty}^{\infty}\left|u_{1}, u_{2}\right|^{\frac{\alpha}{2}-(1+P)} \exp \left(\left|C_{T}^{(1)}\left(u_{1}, u_{2}\right)-C_{T}^{(2)}\left(u_{1}, u_{2}\right)\right|\right. \\
& \left.-C_{T}^{(2)}\left(u_{1}, u_{2}\right)\right) d u_{1} d u_{2}<\infty
\end{aligned}
$$

and this completes our proof. 


\section{REFERENCES}

[1] G. Samorodnitsky, and M.S. Taqqu, "Stable Non-Gaussian Random Processes: Stochastic Models with Infinite Variance", Chapman \& Hall, 1994.

[2] V.M. Zolotarev, and V.V. Uchaikin, "Chance and stability distributions and their applications", VSP, Utrecht, 1999.

[3] M.A. Ghazal, "On the average Spectral of contnuous-time processes costruction and estimation", J. Comb. Inf. Sys. Sci., vol. 29, Nos 1-4, pp. 81-91, 2005.

[4] M.A. Ghazal and A. Mutwalli Aly, "Time series with Poisson point process", J. Appl. Math. Comput., vol. 150, pp. 149-175, 2004.

[5] M.A. Ghazal, and E.A. Farag, "Some properties of the continuous expanded finite fourier transform", Statistica, vol. 3, pp. 577-584, 2000 .
[6] S.H. Combanis, "Two classes of self similar stable processes with stationary increments", J. Stochastic Proc. Appl., vol. 32, pp. 305329, 1989.

[7] Y. Hosoya, "Harmonizable stable processes", Z. Wahrscheinlichkei-tstheorie verw. Gebiete, vol. 60, pp. 517-533, 1982.

[8] C.D. Hardine, "On the spectral representation of symmetric stable processes", J. Multivariate Anal., vol. 12, pp. 385-401, 1982.

[9] E. Masry, and S.H. Cambanis, "Spectraldensity estimation for stationary stable processes", J. Stochastic Proc. App., vol. 18, pp. $1-31,1984$.

(C) M.A. Ghazal; Licensee Bentham Open.

This is an open access article licensed under the terms of the Creative Commons Attribution Non-Commercial License (http://creativecommons.org/licenses/by$\mathrm{nc} / 3.0 /$ ), which permits unrestricted, non-commercial use, distribution and reproduction in any medium, provided the work is properly cited. 